\title{
Guaranteed Cost Control for Uncertain Distributed Delay System with Neutral Type
}

\author{
Yuyan Zhang*, Dan Zhao, Yan Zhao, and Na Zhang \\ Department of Automatic Control Engineering, Shenyang Institute of Engineering, \\ 110136 Shenyang, China \\ \{zhangyy, zhaodan, zhaoyan\} @sie.edu.cn
}

\begin{abstract}
This paper investigates the problem of guaranteed cost control for a class of uncertain distributed delay systems with neutral type. A sufficient condition for the solvability of this problem is obtained. A novel LyapunovKrasovskii functional is constructed to reduce conservatism of the criterion in form of LMIs. Some mathematical techniques are utilized flexibly. Especially, the exchange of the order of repeated integral is required. Based the criterion, the control gain and the guaranteed cost are obtained. A numerical example is provided to illustrate the effectiveness of the proposed design methods.
\end{abstract}

Keywords: Distributed delay system with neutral type, Guaranteed cost control, Linear matrix inequality (LMI).

\section{Introduction}

Neutral delay systems are the general form of delay system and contain delays on the derivatives of some system variables. There are many physical examples for neutral delay system in practical systems (see, e.g., [1,2,8,11]). In recent years, the stability analysis and robust guaranteed cost control problems of neutral delay system have been considered extensively[1,2,5,8,11,12]. Different from some existing results, Lien[1] introduces a positive constant $\omega$ to obtain the corresponding LMIs using Lyapunov--Krasobskii theory and Leibniz--Newton formulae. An LMI optimization approach is proposed to find the robust non-fragile guaranteed cost control and used to minimize the guaranteed cost. $\mathrm{Xu}$ in [11] are concerned with the problem of nonfragile positive real control for uncertain neutral system with invariant delays in state. For both the cases with additive and multiplicative control uncertainties, sufficient conditions for the existence of the controllers are given in terms of LMIs.

On the other hand, with the increasing of the number of summands in a system equation and the decreasing of differences between neighboring argument values, systems with distributed delays will arise. Distributed delays can also be found in the

This work is supported by the Natural Science Foundation of China under Grant No. 60972164, 60904101, 60904046 and the Science and Technology Program of Shenyang under Grant No. F11-264-1-70, F10-205-1-80. 
modeling of feeding systems and combustion chambers in a liquid monopropellant rocket motor with pressure feeding [3,4]. Therefore, many efforts have been made for the distributed delays systems[5,6,7].

To the best of the authors' knowledge, there are a few notes on the problem of guaranteed cost control for distributed delay systems with neutral type, which motivates the present study. In view of the importance of the choice of an appropriate Lyapunov-Krasovskii functional for deriving good stability criteria for delay system, a class of special forms of Lyapunov-Krasovskii functionals are constructed to lead to simpler and less conservative sufficient conditions. Simultaneously, some mathematical techniques are applied flexibly. Finally, a numerical example is provided to illustrate the effectiveness of the proposed design methods.

\section{Problem Statement and Preliminaries}

Consider the following uncertain distributed delay systems with neutral type

$$
\begin{aligned}
\dot{x}(t) & =[A+\Delta A(t)] x(t)+\left[A_{\sigma}+\Delta A_{\sigma}(t)\right] x(t-\sigma)+\left[A_{\eta}+\Delta A_{\eta}(t)\right] \dot{x}(t-\eta) \\
& +\left[A_{\delta}+\Delta A_{\delta}(t)\right] \int_{t-\delta}^{t} x(s) d s+[B+\Delta B(t)] u(t) \\
x(t) & =\phi(t), t \in[-l, 0]
\end{aligned}
$$

where $x(t) \in \mathfrak{R}^{n}$ and $u(t) \in \mathfrak{R}^{m}$ are the state and input of system, respectively. $A, A_{\sigma}, A_{\eta}, A_{\delta}$, and $B$ are known constant matrices of appropriate dimensions. $\phi(t)$ is the initial condition. $\sigma, \eta$, and $\delta$ are the time delays, $l=\max \{\sigma, \eta, \delta\}$. Time-varying parametric uncertainties $\Delta A(t), \Delta A_{\sigma}(t), \Delta A_{\eta}(t), \Delta A_{\delta}(t)$ and $\Delta B(t)$ are assumed to satisfy

$$
\begin{aligned}
& {\left[\begin{array}{lllll}
\Delta A(t) & \Delta A_{\sigma}(t) & \Delta A_{\eta}(t) & \Delta A_{\delta}(t) & \Delta B(t)
\end{array}\right] } \\
= & D F(t)\left[\begin{array}{lllll}
E_{1} & E_{\sigma} & E_{\eta} & E_{\delta} & E_{2}
\end{array}\right]
\end{aligned}
$$

where matrices $D, E_{1}, E_{\sigma}, E_{\eta}, E_{\delta}$, and $E_{2}$ are constant matrices of appropriate dimensions, and $F(t)$ is the unknown matrix function satisfying $F^{T}(t) F(t) \leq I, \forall t \geq 0$.

Construct the following control law

$$
u(t)=-K x(t)
$$

where $K \in \mathfrak{R}^{m \times n}$ is the control gain to be designed, the resulting closed-loop uncertain neutral system is obtained, 


$$
\begin{aligned}
\dot{x}(t) & =\left[A-B K+D F(t)\left(E_{1}-E_{2} K\right)\right] x(t)+\left[A_{\sigma}+D F(t) E_{\sigma}\right] x(t-\sigma) \\
& +\left[A_{\eta}+D F(t) E_{\eta}\right] \dot{x}(t-\eta)+\left[A_{\delta}+D F(t) E_{\delta}\right] \int_{t-\delta}^{t} x(s) d s
\end{aligned}
$$

Define the following quadratic cost function

$$
J=\int_{0}^{\infty}\left[x^{T}(t) S_{1} x(t)+u^{T}(t) S_{2} u(t)\right] d t
$$

where $S_{1} \in \mathfrak{R}^{n \times n}$ and $S_{2} \in \mathfrak{R}^{m \times m}$ are two given symmetric positive definite matrices.

The objective of this note is to design a control (3) and determine a scalar $J_{u}$ satisfying the following two conditions

(a) the closed-loop system (4) is asymptotically stable,

(b) $J \leq J_{u}$.

If the aforementioned control gain $K$ and constant $J_{u}$ exist, control (3) is the guaranteed cost control and $J_{u}$ is the guaranteed cost for the considered system.

The following lemma is used for the proof of the main results.

Lemma 2.1 Let $Z, X, S$ and $Y$ be matrices of appropriate dimensions. Assume that $Z$ is symmetric and $S^{T} S \leq I$, then

$$
Z+X S Y+Y^{T} S^{T} X^{T}<0
$$

if and only if there exists a scalar $\varepsilon>0$ satisfying

$$
Z+\varepsilon X X^{T}+\mathcal{E}^{-1} Y^{T} Y=Z+\varepsilon^{-1}(\varepsilon X)(\varepsilon X)^{T}+\mathcal{E}^{-1} Y^{T} Y<0 .
$$

\section{Main Result}

Theorem 3.1 Consider system (1) with (2) and (3). If there exist positive numbers $\mathcal{E}$, $\tau$, some symmetric positive definite matrices $Q, \bar{H}_{1}, \bar{H}_{2}$, and matrix $X$ such that the following LMIs hold 


$$
\begin{aligned}
& \Xi=\left[\begin{array}{ccccccccc}
\Xi_{11} & A_{\sigma} Q & A_{\eta} & A_{\delta} Q & Q A^{T}-X^{T} B^{T} & Q & -X^{T} & \varepsilon D & \Xi_{19} \\
* & -\bar{H}_{1} & 0 & 0 & Q A_{\sigma}^{T} & 0 & 0 & 0 & Q E_{\sigma}^{T} \\
* & * & -I_{n} & 0 & A_{\eta}^{T} & 0 & 0 & 0 & E_{\eta}^{T} \\
* & * & * & -\bar{H}_{2} & Q A_{\delta}^{T} & 0 & 0 & 0 & Q E_{\delta}^{T} \\
* & * & * & * & -I_{n} & 0 & 0 & \varepsilon D & 0 \\
* & * & * & * & * & -S_{1}^{-1} & 0 & 0 & 0 \\
* & * & * & * & * & * & -S_{2}^{-1} & 0 & 0 \\
* & * & * & * & * & * & * & -\varepsilon I_{n} & 0 \\
* & * & * & * & * & * & * & * & -\varepsilon I_{n}
\end{array}\right]<0 \\
& \Gamma=\left[\begin{array}{ccc}
-I_{n}+\tau E_{\eta}^{T} E_{\eta} & A_{\eta}^{T} & 0 \\
* & -I_{n} & D \\
* & * & -\tau I_{n}
\end{array}\right]<0
\end{aligned}
$$

Then control (3) with $K=X Q^{-1}$ is the guaranteed cost control of system (1) with the following guaranteed cost

$$
\begin{aligned}
J_{u} & =\phi^{T}(0) Q^{-1} \phi(0)+\int_{-\sigma}^{0} \phi^{T}(s) Q^{-1} \bar{H}_{1} Q^{-1} \phi(s) d s+\int_{-\eta}^{0} \dot{\phi}^{T}(s) \dot{\phi}(s) d s \\
& +\int_{-\delta}^{0}\left[\int_{s}^{0} \phi^{T}(\theta) d \theta\right] Q^{-1} \bar{H}_{2} Q^{-1}\left[\int_{s}^{0} \phi(\theta) d \theta\right] d s \\
& +\int_{0}^{\delta} d s \int_{-s}^{0}(\theta+s) \phi^{T}(\theta) Q^{-1} \bar{H}_{2} Q^{-1} \phi(\theta) d \theta
\end{aligned}
$$

where $\Xi_{11}=A Q+Q A^{T}-B X-X^{T} B^{T}+\bar{H}_{1}+\delta^{2} \bar{H}_{2}, \Xi_{19}=Q E_{1}^{T}-X^{T} E_{2}^{T}$.

Proof: Choose $P=Q^{-1}, H_{1}=Q^{-1} \bar{H}_{1} Q^{-1}, H_{2}=Q^{-1} \bar{H}_{2} Q^{-1}$, and construct the following Lyapunov functional

$$
V(x(t), t)=V_{1}(x(t))+V_{2}(x(t))+V_{3}(x(t))+V_{4}(x(t))+V_{5}(x(t))
$$

where

$$
\begin{aligned}
& V_{1}(x(t))=x^{T}(t) \operatorname{Px}(t), V_{2}(x(t))=\int_{t-\sigma}^{t} x^{T}(s) H_{1} x(s) d s, \\
& V_{3}(x(t))=\int_{t-\eta}^{t} \dot{x}^{T}(s) \dot{x}(s) d s, V_{4}(x(t))=\int_{t-\delta}^{t}\left[\int_{s}^{t} x^{T}(\theta) d \theta\right] H_{2}\left[\int_{s}^{t} x(\theta) d \theta\right] d s, \\
& V_{5}(x(t))=\int_{0}^{\delta} d s \int_{t-s}^{t}(\theta-t+s) x^{T}(\theta) H_{2} x(\theta) d \theta
\end{aligned}
$$


Obviously, $V(x(t), t)>0$ for all $x(t) \neq 0$. The time derivative of $V(x(t), t)$ along the trajectories of system (1) with control (3) is given

$$
\dot{V}(x(t), t)=\dot{V}_{1}(x(t))+\dot{V}_{2}(x(t))+\dot{V}_{3}(x(t))+\dot{V}_{4}(x(t))+\dot{V}_{5}(x(t))
$$

where

$$
\begin{aligned}
\dot{V}_{1}(x(t))= & 2 x^{T}(t) P\left[\left(A-B K+D F(t)\left(E_{1}-E_{2} K\right)\right) x(t)\right. \\
& +\left(A_{\sigma}+D F(t) E_{\sigma}\right) x(t-\sigma)+\left(A_{\eta}+D F(t) E_{\eta}\right) \dot{x}(t-\eta) \\
& +\left(A_{\delta}+D F(t) E_{\delta}\right) \int_{t-\delta}^{t} x(s) d s \\
\dot{V}_{2}(x(t))= & x^{T}(t) H_{1} x(t)-x^{T}(t-\sigma) H_{1} x(t-\sigma), \\
\dot{V}_{3}(x(t))= & \dot{x}^{T}(t) \dot{x}(t)-\dot{x}^{T}(t-\eta) \dot{x}(t-\eta) \\
= & {\left[\left(A-B K+D F(t)\left(E_{1}-E_{2} K\right)\right) x(t)+\left(A_{\sigma}+D F(t) E_{\sigma}\right) x(t-\sigma)\right.} \\
& \left.+\left(A_{\eta}+D F(t) E_{\eta}\right) \dot{x}(t-\eta)+\left(A_{\delta}+D F(t) E_{\delta}\right) \int_{t-\delta}^{t} x(s) d s\right]^{T} \\
& \times\left[\left(A-B K+D F(t)\left(E_{1}-E_{2} K\right)\right) x(t)+\left(A_{\sigma}+D F(t) E_{\sigma}\right) x(t-\sigma)\right. \\
& \left.\left.+\left(A_{\eta}+D F(t) E_{\eta}\right) \dot{x}(t-\eta)+\left(A_{\delta}+D F(t) E_{\delta}\right)\right]_{t-\delta}^{t} x(s) d s\right] \\
& -\dot{x}^{T}(t-\eta) \dot{x}(t-\eta), \\
\dot{V}_{4}(x(t))= & 2 \int_{t-\delta}^{t}(\theta-t+\delta) x^{T}(t) H_{2} x(\theta) d \theta \\
& -\left[\int_{t-\delta}^{t} x^{T}(\theta) d \theta\right] H_{2}\left[\int_{t-\delta}^{t} x(\theta) d \theta\right] \\
\leq & \int_{t-\delta}^{t}(\theta-t+\delta) x^{T}(t) H_{2} x(t) d(\theta-t+\delta) \\
& +\int_{t-\delta}^{t}(\theta-t+\delta) x^{T}(\theta) H_{2} x(\theta) d \theta \\
& -\left[\int_{t-\delta}^{t} x^{T}(\theta) d \theta\right] H_{2}\left[\int_{t-\delta}^{t} x(\theta) d \theta\right] \\
\dot{V}_{5}(x(t))= & \frac{1}{2} \delta^{2} x^{T}(t) H_{2} x(t)-\int_{t-\delta}^{t}(\theta-t+\delta) x^{T}(\theta) H_{2} x(\theta) d \theta . \\
& \frac{1}{2} \delta^{2} x^{T}(t) H_{2} x(t)+\int_{t-\delta}^{t}(\theta-t+\delta) x^{T}(\theta) H_{2} x(\theta) d \theta \\
t & \left.x^{T}(\theta) d \theta\right] H_{2}\left[\int_{t-\delta}^{t} x(\theta) d \theta\right], \\
& {\left[\int_{t}(\theta)\right.} \\
&
\end{aligned}
$$


Therefore, it follows from (10) that

$$
\begin{aligned}
\dot{V}(x(t), t)+x^{T}(t) S_{1} x(t)+u^{T}(t) S_{2} u(t)= & \dot{V}(x(t), t)+x^{T}(t) S_{1} x(t) \\
& +x^{T}(t) K^{T} S_{2} K x(t) \\
& \leq \xi^{T} \mathrm{Y} \xi
\end{aligned}
$$

where

$$
\begin{aligned}
& \xi^{T}=\left[\begin{array}{llll}
x^{T}(t) & x^{T}(t-\sigma) & \dot{x}^{T}(t-\eta) & \int_{t-\delta}^{t} x^{T}(s) d s
\end{array}\right], \\
& \mathrm{Y}=\Omega+G_{1} G_{1}^{T}+G_{2} S_{1} G_{2}^{T}+G_{3} S_{2} G_{3}^{T}, \\
& \Omega=\left[\begin{array}{cccc}
\Omega_{11} & P\left[A_{\sigma}+D F(t) E_{\sigma}\right] & P\left[A_{\eta}+D F(t) E_{\eta}\right] & P\left[A_{\delta}+D F(t) E_{\delta}\right] \\
* & -H_{1} & 0 & 0 \\
* & * & -I_{n} & 0 \\
* & * & * & -H_{2}
\end{array}\right], \\
& \Omega_{11}=P\left[A-B K+D F(t)\left(E_{1}-E_{2} K\right)\right]+\left[A-B K+D F(t)\left(E_{1}-E_{2} K\right)\right]^{T} P \\
& +H_{1}+\delta^{2} H_{2} \\
& G_{1}=\left[\begin{array}{c}
(A-B K)^{T}+\left(E_{1}-E_{2} K\right)^{T} F^{T}(t) D^{T} \\
A_{\sigma}^{T}+E_{\sigma}^{T} F^{T}(t) D^{T} \\
A_{\eta}^{T}+E_{\eta}^{T} F^{T}(t) D^{T} \\
A_{\delta}^{T}+E_{\delta}^{T} F^{T}(t) D^{T}
\end{array}\right], G_{2}=\left[\begin{array}{c}
I_{n} \\
0 \\
0 \\
0
\end{array}\right], G_{3}=\left[\begin{array}{c}
-K^{T} \\
0 \\
0 \\
0
\end{array}\right] .
\end{aligned}
$$

Define

$$
\overline{\mathrm{Y}}=\left[\begin{array}{cccc}
\Omega & G_{1} & G_{2} & G_{3} \\
* & -I_{n} & 0 & 0 \\
* & * & -S_{1}^{-1} & 0 \\
* & * & * & -S_{2}^{-1}
\end{array}\right]
$$

Pre- and post-multiplying the matrix $\overline{\mathrm{Y}}$ in (12) by $U^{T}$ and $U$, where

$$
U=\operatorname{diag}\left\{P^{-1}, P^{-1}, I_{n}, P^{-1}, I_{n}, I_{n}, I_{m}\right\}=\operatorname{diag}\left\{Q, Q, I_{n}, Q, I_{n}, I_{n}, I_{m}\right\},
$$

and substituting $K Q, Q H_{1} Q$, and $Q H_{2} Q$ with $X, \bar{H}_{1}$, and $\bar{H}_{2}$, respectively, the following matrix is obtained 


$$
\Theta=\left[\begin{array}{cccc}
\bar{\Omega} & \bar{G}_{1} & \bar{G}_{2} & \bar{G}_{3} \\
* & -I_{n} & 0 & 0 \\
* & * & -S_{1}^{-1} & 0 \\
* & * & * & -S_{2}^{-1}
\end{array}\right]
$$

where

$$
\begin{aligned}
& \bar{\Omega}=\left[\begin{array}{cccc}
\bar{\Omega}_{11} & {\left[A_{\sigma}+D F(t) E_{\sigma}\right] Q} & A_{\eta}+D F(t) E_{\eta} & {\left[A_{\delta}+D F(t) E_{\delta}\right] Q} \\
* & -\bar{H}_{1} & 0 & 0 \\
* & * & -I_{n} & 0 \\
* & * & * & -\bar{H}_{2}
\end{array}\right], \\
& \bar{\Omega}_{11}=A Q-B X+D F\left(E_{1} Q-E_{2} X\right)+Q A^{T}-X^{T} B^{T} \\
& +\left(Q E_{1}^{T}-X^{T} E_{2}^{T}\right) F^{T}(t) D^{T}+\bar{H}_{1}+\delta^{2} \bar{H}_{2}, \\
& \bar{G}_{1}=\left[\begin{array}{c}
Q A^{T}-X^{T} B^{T}+\left(Q E_{1}^{T}-X^{T} E_{2}^{T}\right) F^{T}(t) D^{T} \\
Q A_{\sigma}^{T}+Q E_{\sigma}^{T} F^{T}(t) D^{T} \\
A_{\eta}^{T}+E_{\eta}^{T} F^{T}(t) D^{T} \\
Q A_{\delta}^{T}+Q E_{\delta}^{T} F^{T}(t) D^{T}
\end{array}\right], \bar{G}_{2}=\left[\begin{array}{c}
Q \\
0 \\
0 \\
0
\end{array}\right], \bar{G}_{3}=\left[\begin{array}{c}
-X^{T} \\
0 \\
0 \\
0
\end{array}\right] .
\end{aligned}
$$

It is obvious that

$$
\begin{aligned}
\Theta= & {\left[\begin{array}{ccccccc}
\Xi_{11} & A_{\sigma} Q & A_{\eta} & A_{\delta} Q & Q A^{T}-X^{T} B^{T} & Q & -X^{T} \\
* & -\bar{H}_{1} & 0 & 0 & Q A_{\sigma}^{T} & 0 & 0 \\
* & * & -I_{n} & 0 & A_{\eta}^{T} & 0 & 0 \\
* & * & * & -\bar{H}_{2} & Q A_{\delta}^{T} & 0 & 0 \\
* & * & * & * & -I_{n} & 0 & 0 \\
* & * & * & * & * & -S_{1}^{-1} & 0 \\
* & * & * & * & * & * & -S_{2}^{-1}
\end{array}\right] } \\
& +\Lambda^{T} F(t) \$+\$^{T} F^{T}(t) \Lambda
\end{aligned}
$$

where

$$
\begin{aligned}
& \Lambda=\left[\begin{array}{llllllll}
D^{T} & 0 & 0 & 0 & D^{T} & 0 & 0
\end{array}\right], \\
& \$=\left[\begin{array}{llllllll}
E_{1} Q-E_{2} X & E_{\sigma} Q & E_{\eta} & E_{\delta} Q & 0 & 0 & 0
\end{array}\right] .
\end{aligned}
$$


By Lemma 2.1 and Schur complement formula, the condition $\Xi<0$ in (6) is equivalent to $\Theta<0$ in (14). According to (12), (13), and (14), $\Theta<0$ is equivalent to $\bar{Y}<0$. Applying Schur complement formula to $\bar{Y}<0$, one can obtain $\mathrm{Y}<0$ in (11). From the condition $\mathrm{Y}<0$ in (11), there exists a constant $\rho>0$, such that

$$
\dot{V}(x(t), t) \leq-\not \| x\left(t \|_{2}^{2}\right.
$$

Another, by Schur complement formula, $\Gamma<0$ in (7) is equivalent to the following one

$$
\left[\begin{array}{cc}
-I_{n}+\tau E_{\eta}^{T} E_{\eta} & A_{\eta}^{T} \\
* & -I_{n}
\end{array}\right]+\tau^{-1}\left[\begin{array}{l}
0 \\
D
\end{array}\right]\left[\begin{array}{ll}
0 & D^{T}
\end{array}\right]<0
$$

that is

$$
\left[\begin{array}{cc}
-I_{n} & A_{\eta}^{T} \\
* & -I_{n}
\end{array}\right]+\tau\left[\begin{array}{c}
E_{\eta}^{T} \\
0
\end{array}\right]\left[\begin{array}{ll}
E_{\eta} & 0
\end{array}\right]+\tau^{-1}\left[\begin{array}{c}
0 \\
D
\end{array}\right]\left[\begin{array}{ll}
0 & D^{T}
\end{array}\right]<0
$$

According to Lemma 2.1, inequality (17) is equivalent to the following one

$$
\left[\begin{array}{cc}
-I_{n} & A_{\eta}^{T} \\
* & -I_{n}
\end{array}\right]+\left[\begin{array}{l}
0 \\
D
\end{array}\right] F(t)\left[\begin{array}{ll}
E_{\eta} & 0
\end{array}\right]+\left[\begin{array}{c}
E_{\eta}^{T} \\
0
\end{array}\right] F^{T}(t)\left[\begin{array}{ll}
0 & D^{T}
\end{array}\right]<0
$$

that is

$$
\left[\begin{array}{cc}
-I_{n} & A_{\eta}^{T}+E_{\eta}^{T} F^{T}(t) D^{T} \\
* & -I_{n}
\end{array}\right]<0
$$

By Schur complement formula, inequality (19) is equivalent to the following one

$$
\left(A_{\eta}+D F(t) E_{\eta}\right)^{T}\left(A_{\eta}+D F(t) E_{\eta}\right)<I_{n}
$$

that is

$$
\left\|A_{\eta}+\Delta A_{\eta}\right\|_{2}<1
$$

This means that system (1) is Lipschitz in the term $\dot{x}(t-\eta(t))$ with Lipschitz constant less than 1 .

Hence, by conditions (9) and (15), and (21), one can conclude that system (1) with (2) and (3) is asymptotically stable. From (11) with $\mathrm{Y}<0$, one can obtain

$$
\begin{aligned}
\int_{0}^{\infty} \dot{V}(x(t), t) d t & =\lim _{t \rightarrow \infty} V(x(t), t)-V(x(0), 0) \\
& \leq-\int_{0}^{\infty}\left[x^{T}(t) S_{1} x(t)+u^{T}(t) S_{2} u(t)\right] d t
\end{aligned}
$$


Therefore,

$$
\lim _{t \rightarrow \infty} V(x(t), t)=0, \int_{0}^{\infty}\left[x^{T}(t) S_{1} x(t)+u^{T}(t) S_{2} u(t)\right] d t \leq V(x(0), 0)=J_{u} .
$$

This completes the proof.

\section{$4 \quad$ Illustrative Example}

In this section, a numerical example is presented to show the validity of the control approach. A three-order system with two inputs is considered with the following parameters

$$
\begin{aligned}
& A=\left[\begin{array}{ccc}
-25.2 & 1.3 & 3.3 \\
11.5 & -3.5 & 2.8 \\
2.9 & -14.1 & -5.1
\end{array}\right], A_{\sigma}=\left[\begin{array}{ccc}
-2.1 & -1.3 & 0.2 \\
1.1 & 0.5 & 0.5 \\
0.7 & -1.6 & 0.1
\end{array}\right], B=\left[\begin{array}{cc}
1.5 & 2.2 \\
-2.3 & -0.1 \\
-3.5 & 1.5
\end{array}\right], \\
& A_{\eta}=\left[\begin{array}{ccc}
0.1 & -0.3 & -0.1 \\
0.1 & 0.5 & -0.1 \\
0.2 & 0.1 & -0.5
\end{array}\right], A_{\delta}=\left[\begin{array}{ccc}
-2.1 & 0.2 & -0.4 \\
0.1 & 0.2 & 0.2 \\
-0.8 & -0.4 & 1.4
\end{array}\right], \sigma=0.1 \text {, } \\
& D=\left[\begin{array}{ccc}
-0.5 & 0.2 & 0.1 \\
0.1 & -0.7 & -0.2 \\
-0.1 & 0.3 & 0.1
\end{array}\right], E_{1}=\left[\begin{array}{ccc}
0.3 & -0.1 & 0.1 \\
0.4 & -0.5 & -0.1 \\
-0.1 & 0.2 & 0.1
\end{array}\right], \eta=0.1 \text {, } \\
& E_{\sigma}=\left[\begin{array}{ccc}
-0.1 & -0.1 & 0.1 \\
-0.1 & 0.2 & 0.1 \\
0.1 & -0.1 & -0.2
\end{array}\right], E_{\eta}=\left[\begin{array}{ccc}
0.1 & 0.1 & -0.1 \\
-0.1 & 0.2 & -0.1 \\
-0.1 & -0.1 & 0.1
\end{array}\right], \delta=0.2 \text {, } \\
& E_{\delta}=\left[\begin{array}{ccc}
-0.2 & -0.2 & 0.3 \\
-0.3 & -0.2 & 0.1 \\
0.1 & 0.4 & 0.4
\end{array}\right], E_{2}=\left[\begin{array}{cc}
-0.6 & -0.3 \\
-0.1 & 0.1 \\
-0.4 & 0.2
\end{array}\right]
\end{aligned}
$$

Let

$$
S_{1}=\operatorname{diag}\{0.5,0.5,0.5\}, S_{2}=\operatorname{diag}\{2,2\}
$$

and the initial condition is given by

$$
\phi(t)=\left[\begin{array}{lll}
-0.1+t & 0.15 & 0.2 t+0.05
\end{array}\right]^{T}
$$

Applying Matlab toolbox [9,10] to solving the LMIs (6) and (7) with above parameters, one can obtain 


$$
\begin{aligned}
& Q=\left[\begin{array}{ccc}
0.0405 & -0.0193 & -0.0007 \\
-0.0193 & 0.0611 & -0.0810 \\
-0.0007 & -0.0810 & 0.1461
\end{array}\right], \varepsilon=0.6067 \text {, } \\
& X=\left[\begin{array}{ccc}
-0.1478 & 0.0268 & -0.2684 \\
-0.0149 & -0.0876 & 0.2976
\end{array}\right], \tau=5.1734 \text {, } \\
& \bar{H}_{1}=\left[\begin{array}{ccc}
0.3369 & -0.1033 & -0.0637 \\
-0.1033 & 0.1822 & -0.0344 \\
-0.0637 & -0.0344 & 0.2582
\end{array}\right] \text {, } \\
& \bar{H}_{2}=\left[\begin{array}{ccc}
7.8186 & -2.3760 & -1.4933 \\
-2.3760 & 4.0091 & -0.0089 \\
-1.4933 & -0.0089 & 5.0074
\end{array}\right] \text {, } \\
& P=\left[\begin{array}{ccc}
60.5751 & 73.8035 & 41.2087 \\
73.8035 & 151.7409 & 84.4717 \\
41.2087 & 84.4717 & 53.8685
\end{array}\right] \text {, } \\
& K=\left[\begin{array}{ccc}
-18.0362 & -29.5139 & -18.2854 \\
4.8911 & 10.7387 & 8.0133
\end{array}\right] \text {, } \\
& H_{1}=\left[\begin{array}{lll}
1.2164 & 1.9843 & 1.1349 \\
1.9843 & 3.8833 & 2.2436 \\
1.1349 & 2.2436 & 1.3066
\end{array}\right] * 10^{3} \text {, } \\
& H_{2}=\left[\begin{array}{lll}
3.0276 & 5.0207 & 2.8767 \\
5.0207 & 9.8562 & 5.7011 \\
2.8767 & 5.7011 & 3.3162
\end{array}\right] * 10^{4}, \\
& J_{u}=10.7543 .
\end{aligned}
$$

\section{Conclusion}

The guaranteed cost control for uncertain distributed delay system with neutral type is complex and challenging. In view of the importance of the choice of an appropriate Lyapunov-Krasovskii functional for deriving good stability criteria for delay system, a special form of Lyapunov-Krasovskii functional is constructed to lead to simpler and less conservative sufficient conditions. Simultaneously, some mathematical techniques are applied flexibly. Especially, the order of repeated integral is 
exchanged. Based the criterion, the control gain and the guaranteed cost are obtained.Finally, the numerical example has shown the validity of the present control approach.

\section{References}

1. Lien, C.H.: Non-fragile guaranteed cost control for uncertain neutral dynamic systems with time-varying delays in state and control input. Chaos, Solitons \& Fractals 31(4), 889-899 (2007)

2. Ivanevescu, D., Niculescu, S.I., Dugard, L., Dion, J.M., Verriest, E.I.: On delay-dependent stability for linear neutral systems. Automatica 39(2), 255-261 (2003)

3. $\mathrm{Xu}, \mathrm{S}$., Chen, T.: An LMI approach to the $H_{\infty}$ filter design for uncertain system with distributed delays. IEEE Trans. on Circuits and Systems-II 51(4), 195-201 (2004)

4. Zheng, F., Frank, P.M.: Robust control of uncertain distributed delay systems with application to the stabilization of combustion in rocket motor chambers. Automatica 38(3), 487-497 (2002)

5. Xu, S.Y., Lam, J., Chen, T.W., Zou, Y.: A delay-dependent approach to robust filtering for uncertain distributed delay systems. IEEE Transactions on Signal Processing 53(10), 3764-3772 (2005)

6. Cheng, Y.C., Hwang, C., Chen, C.T.: Evaluation of quadratic cost functionals for a class of distributed-delay systems. IET Control Theory Applications 1(1), 313-319 (2007)

7. Yuan, K., Li, H.X., Cao, J.D.: Robust stabilization of the distributed parameter system with time delay via fuzzy control. IEEE Transactions on Fuzzy Systems 16(3), 567-584 (2008)

8. Park, J.H.: Robust guaranteed cost control for uncertain linear differential systems of neutral type. Applied Mathematics and Computation 140(2-3), 523-535 (2003)

9. Gahinet, P., Nemirovski, A., Laub, A.J., Chilali, M.: LMI control toolbox user's guide. The Math Works Inc., Natick Mass (1995)

10. Boyd, S., Ghaoui, L.E., Feron, E., Balakrishnan, V.: Linear Matrix Inequalities in Systems and Control Theory. SIAM, Philadelphia (1994)

11. Xu, S.Y., Lam, J., Wang, J.L., Yang, G.H.: Non-fragile positive real control for uncertain linear neutral delay systems. System \& Control Letters 52(1), 59-74 (2004)

12. Lien, C.H., Yu, K.W.: Non-fragile $H_{\infty}$ control for uncertain neutral systems with timevarying delays via the LMI optimization approach. IEEE Transactions on Systems, Man, Cybernetics-Part B: Cybernetics 37(2), 493-499 (2007) 